\title{
A Probabilistic Approach for the Evaluation of Fault Detection Schemes in Microgrids
}

\author{
R. Eslami \\ Department of Electrical \\ Engineering \\ Amirkabir University of \\ Technology \\ Tehran, Iran \\ reza.eslami@aut.ac.ir
}

\author{
S. H. H. Sadeghi \\ Department of Electrical \\ Engineering \\ Amirkabir University of \\ Technology \\ Tehran, Iran \\ sadeghi@aut.ac.ir
}

\author{
H. Askarian Abyaneh \\ Department of Electrical \\ Engineering \\ Amirkabir University of \\ Technology \\ Tehran, Iran \\ askarian@aut.ac.ir
}

\begin{abstract}
An important challenge in protection of a microgrid is the process of fault detection, considering the uncertainties in its topologies. Equally important is the evaluation of proposed methods as their incorrect performances could result in unreasonable power outages. In this paper, a new fault detection and characterization method is introduced and evaluated subject to the uncertainties of network topologies. The features of threephase components together with the positive, negative and zero sequences of current and voltage waveforms are derived to detect the occurrence of a fault, its location, type and the engaged phases. The proposed method is independent of the microgrid topology. To evaluate the performance of the proposed method in various network topologies, a Monte Carlo scheme is developed. This is done by computing the expected energy not-supplied reliability index and the percentage of successful performance of the fault detection. Simulation results show that the proposed method can detect faults in various microgrid topologies with a very high degree of accuracy.
\end{abstract}

Keywords-fault detection; reliability; network sequences; $S$ transform; signal energy

\section{INTRODUCTION}

Despite the benefits counted for the microgrids, their designs remained at the laboratory level because of various technical reasons. One of the most important technical issues is the challenge of protecting these networks [1]. The dynamic behavior of microgrids in which a distributed generation (DG) is connected or disconnected from the network at any time and also the performance of the microgrids in both normal and isolated modes create the most important problems in protecting these networks [2]. The dynamic behavior of microgrids leads to diversity in magnitudes and the current directions which encounters the fault detection procedure with major problems. This issue signifies the necessity of studies in the field of determining the appropriate protection method for the microgrids [3]. The authors in [4-6] have introduced the protection challenges of microgrids. In $[3,4,7,8]$, authors categorized the various methods for solving this problem. These methods included voltage analysis, harmonic analysis, wavelet analysis, and S analysis. In this respect, authors in [9] suggested monitoring output voltage of distributed generations and transferring them from abc axis to $\mathrm{dq}$ axis and then determining threshold value in dq axis for detecting fault in the microgrids. In [10], authors detected faults using created spectrums from the harmonic impedance and their comparison by the central server. Using wavelet transform analysis and $\mathrm{S}$ analysis was suggested in $[11,12]$ and deriving signal energy was investigated in [13]. All these studies considered only a certain topology for the microgrid so the effectiveness of these methods may face challenge in other topologies. In [14], the fault is detected by monitoring the THD of the output voltage of distributed generations and its comparison with a threshold value. In [15], authors used harmonic analysis for fault detection and the proportion of zero sequence current to positive sequence current in the fifth harmonic is used and only single phase to ground faults could be detected by it. Lack of attention to the dynamic topology of microgrids, difficulty in detecting various faults (regular faults and high impedance) and inability to identify the transient states from the persistent faults were examples of problems seen in the previous studies. Thus, in this paper a new method is suggested for fault detection in microgrids and is evaluated by means of Monte Carlo Simulation. S-Transform, positive, negative and zero sequences of current and voltage waveforms are used to detect faults. The presented method can respond to all dynamic topologies of microgrids. As it was mentioned, the new method is evaluated using Monte Carlo Simulation. To do this, the performance accuracy of this method in detecting various faults is evaluated by means of Expected Energy Not-Supplied reliability index (EENS) and percentage of successful performances. Validation results of the suggested method for the persistent and transient faults properly show that this method has acted very well with very high reliability in detecting various faults in different topologies. In addition, this method can distinguish the difference between the persistent faults and transient states of microgrids. 


\section{PROBLEM STATEMENT}

Due to the short lines in microgrids, the fault occurrence in a point of network can lead to disturbance in other points of the network. To show this, Figure 1 is presented. Based on occurred fault in point $\mathrm{F}$ of Figure 1, Figure 2 shows the current signals seen in the various line modules in the network. As seen in Figure 2, by a fault occurring at F, the current waveform of the faulted line is in overlap with the current waveforms of the sidelines. This issue bears difficulty in detecting the exact location of fault using traditional methods. Meanwhile, this issue which the previous studies only emphasized the detection of fault occurrence is not true because inaccuracy in determining the fault location and fault type can lead to unnecessary switching in the safe lines and as a result reduction in protection system reliability.

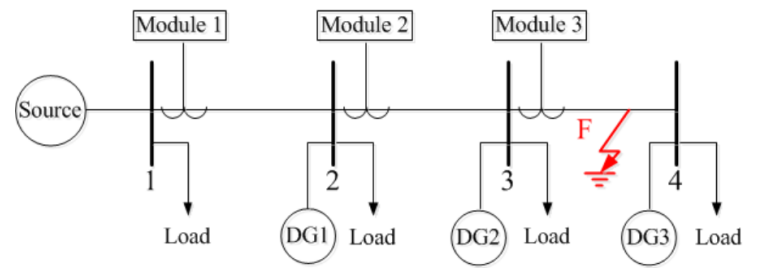

Fig. 1. A sample microgrid

Another issue that challenged the previous microgrid protection methods is their inability to detect different fault types in the network. As mentioned before, short circuit current has high diversity in microgrids. The low amount of this current even encounters challenges of detecting regular faults. However, high impedance faults are of the important possible faults in the electricity networks in which the fault current produced is very low [16]. Therefore, this type of faults requires very accurate methods for detection. This issue caused the authors in [16] to present a new relay design for detecting these types of faults. The new presented relay in [16] can only detect the high impedance faults and neglects the low impedance faults. Therefore, it is obvious that to increase reliability, protection systems have to be designed in such a way that they could effectively protect the network in the all operational structures. Meanwhile, protection has to be able to detect various possible faults (not one special fault) plus their exact locations by using various specifications of turbulent signals.

\section{INTRODUCTION AND EVALUATION OF THE PROPOSED FAULT DETECTION METHOD}

\section{A. Required Substructure for the Implementation of the Proposed Method}

Figure 3 shows the required context for implementing the proposed method in this paper for fault detection in microgrids. As shown in Figure 3, fault detection modules $(m=1: M)$ are installed at the beginning of network lines which have connection with a central server through communication links. The modules continuously take samples from voltage and current signals and deal with the proposed fault detection method to determine the occurrence of a fault, its type, and the line within it occurs. It is worth noting that in order to increase the accuracy of fault detection, the proposed strategy, for detection of fault occurrence, fault location, fault type and the engaged phases, is to use standard deviation (STD) and signal energy obtained from the analysis of positive, negative and zero sequences and meanwhile three-phase components of voltage and current signal. This paper suggests using Stransform to calculate the STD and energy of voltage and current signals.

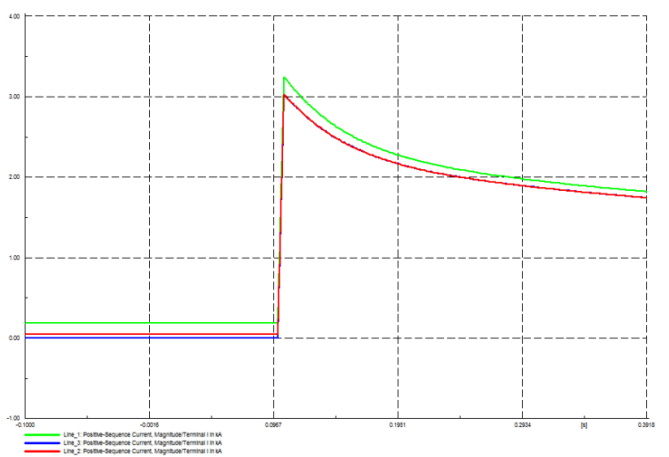

Fig. 2. Fault current signals in different modules of Figure 1 for fault $F$

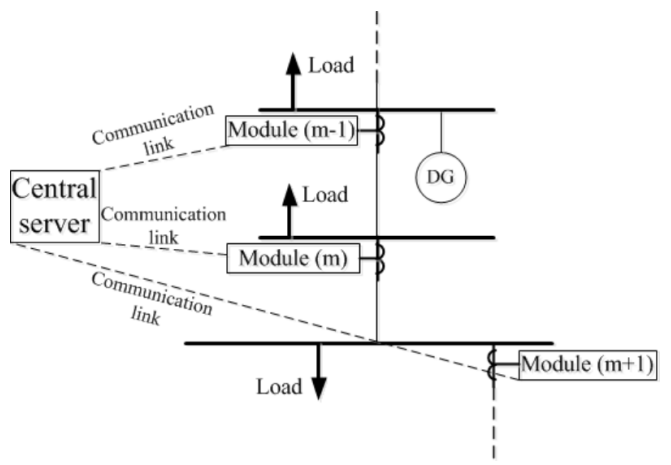

Fig. 3. The required platform for the implementation of the proposed method

\section{B. Fault Detection Process and its Validation Using Monte Carlo}

Since the parameters and uncertainties vary through time, sequential Monte Carlo simulation is used to implement the proposed method. Details have been presented in [17]. During simulation, the intended reliability indices are evaluated due to the possibility of fault occurrence in various network lines and dynamic behavior of microgrid topology. In this respect, implementation flowchart has been shown in Figure 4. As shown, seven subroutines have been considered for implementing the suggested. It is worth noting that the performance accuracy of the proposed method has been evaluated in these subroutines based on the occurrence of permanent faults and transient distortions (including lines switching on and DGs switching on or off).

Subroutine 1: Fixing faulty elements

Step 1: Check whether the line is fixed due to repair rate. 
Step 2: If a line had been fixed, it should be switched on.

Step 3: Implement changes of microgrid topology in the network.

Step 4: If the network topology is changed go to subroutine 2; otherwise go to subroutine 3 .

Subroutine 2: Simulation of transient state.

Step 5: Simulate the transient state as a result of topology change (repaired lines switching on or DGs switching on or off) and then go to subroutine 4 .

Subroutine 3: Random numbers for determining the faulty lines.

Step 6: Compare generated random numbers with the failure rate of lines, if there is a fault in a line, go to Step 7; otherwise go to the step 1 in the next simulation.

Subroutine 4: Fault detection

Step 7: Sample three-phase current and voltage in different points of the network. Signals are sampled by interval time of T and total sampling number being $\mathrm{N}$.

Step 8: Calculate positive, negative and zero sequences based on three-phase components for sampled signals.

Step 9: Apply discrete S-transform to different signals as follows,

$$
S_{u, q}^{m}\left[l T, \frac{n}{N T}\right]=\sum_{k=1}^{N} u_{q}^{m}(k T) \cdot \omega\left((k-l) T, \frac{n}{N T}\right) \cdot \exp \left(\frac{-j 2 \pi k n}{N}\right)
$$

in which $\omega$ is a running Gaussian function window, $m=1: M, q=S e q_{0}, \operatorname{Seq}_{1}, \mathrm{Seq}_{2}, a, b, c$ and $u=i, v ; i$ and $v$ indicate the current and voltage signals respectively.

Step 10: S-matrix, $\mathrm{S}_{u, q}^{m}$, is the S-transform output as (2),

$$
S_{u, q}^{m}\left[l T, \frac{n}{N T}\right]=\left(\begin{array}{cccc}
S_{u, q}^{m}\left[T, \frac{1}{N T}\right] & S_{u, q}^{m}\left[T, \frac{2}{N T}\right] & \cdots & S_{u, q}^{m}\left[T, \frac{1}{T}\right] \\
S_{u, q}^{m}\left[2 T, \frac{1}{N T}\right] & S_{u, q}^{m}\left[2 T, \frac{2}{N T}\right] & \cdots & S_{u, q}^{m}\left[2 T, \frac{1}{T}\right] \\
S_{u, q}^{m}\left[3 T, \frac{1}{N T}\right] & S_{u, q}^{m}\left[3 T, \frac{2}{N T}\right] & \cdots & S_{u, q}^{m}\left[3 T, \frac{1}{T}\right] \\
\vdots & \vdots & \vdots & \vdots \\
S_{u, q}^{m}\left[N T, \frac{1}{N T}\right] & S_{u, q}^{m}\left[N T, \frac{2}{N T}\right] & \cdots & S_{u, q}^{m}\left[N T, \frac{1}{T}\right]
\end{array}\right) \text { (2) }
$$

Step 11: Calculate the energy Matrix, $\mathrm{E}_{u, q}^{m}$, whose elements are the square of the amplitude of the respective elements in the Smatrix.

Step 12: Calculate the signal energy, $E_{u, q}^{m}$, as follows,

$$
E_{u, q}^{m}=\sum_{e=1}^{N} \sum_{g=1}^{N}\left|S_{u, q}^{m}\left[e T, \frac{g}{N T}\right]\right|^{2}
$$

Step 13: Calculate the standard deviation of signal, $S T D_{u, q}^{m}$, as follows,

$$
S T D_{u, q}^{m}=\sqrt[2]{\frac{\sum_{e=1}^{N} \sum_{g=1}^{N}\left(\left|S_{u, q}^{m}\left[e T, \frac{g}{N T}\right]\right|^{2}-\left(\frac{\sum_{e=1}^{N} \sum_{g=1}^{N}\left|S_{u, q}^{m}\left[e T, \frac{g}{N T}\right]\right|^{2}}{N^{2}}\right)\right)^{2}}{N^{2}}}
$$

Step 14: Compare STD and signal energy values of voltage and current signals for positive, negative and zero sequences with threshold values.

Step 15: If all four values of each sequence at Step 14 are above threshold values, it means a fault happened. Otherwise; no fault occurred. If the last simulation was a result of topology change, go Step 6.

Subroutine 5: Detection of fault location

Step 16: Calculate $K_{1}$ index for modules which detected fault as follows,

$K_{1}=\left[\begin{array}{c}2 \times \overline{E_{i, S e q_{0}}^{1}}+\overline{E_{i, S e q_{1}}^{1}}+\overline{E_{i, S e q_{2}}^{1}} \\ 2 \times \overline{E_{i, S e q_{0}}^{2}}+\overline{E_{i, S e q_{1}}^{2}}+\overline{E_{i, S e q_{2}}^{2}} \\ 2 \times \overline{E_{i, S e q_{0}}^{3}}+\overline{E_{i, S e q_{1}}^{3}}+\overline{E_{i, S e q_{2}}^{3}} \\ \bar{\vdots} \\ 2 \times \overline{E_{i, S e q_{0}}^{L}}+\overline{E_{i, S e q_{1}}^{L}}+\overline{E_{i, S e q_{2}}^{L}}\end{array}\right]$

Step 17: Determine two modules with the largest $\mathrm{K}_{1}$.

Step 18: Determine current sign for these two modules (if the crossing current from a module is entering the line of that module, the sign of sensing current is positive. Otherwise; the sign of sensing current is negative).

Step 19: If current sign of these two modules have the same mark, go Step 20. Otherwise; fault location is between these two modules.

Step 20: If there is another line at current flow path, go Step 21. Otherwise, the fault is at the last line of this path.

Step 21: Determine the next module at current flow path and analyze whether this module detects fault occurrence or not. If it is detected, go Step 22. Otherwise; the fault is at the previous passed line of this path.

Step 22: If current sign of this module changed, fault location is at the line where this module locates. Otherwise, go Step 21.

Subroutine 6: Detection process of fault type

Step 23: Calculate $\mathrm{K}_{2}^{\mathrm{m}^{\prime}}$ and $\mathrm{K}_{3}^{\mathrm{m}^{\prime}}$ indices for module $\left(m=m^{\prime}\right)$ having the highest $K_{1}$ index value as follows,

$$
\begin{aligned}
K_{2}^{m^{\prime}} & =\overline{E_{i, S e q_{0}}^{m^{\prime}}}+\overline{E_{v, S e q_{0}}^{m^{\prime}}} \\
K_{3}^{m^{\prime}} & =\overline{E_{i, S e q_{1}}^{m^{\prime}}}+\overline{\left(1 / E_{v, S e q_{1}}^{m^{\prime}}\right)}+\overline{E_{i, S e q_{2}}^{m^{\prime}}}+\overline{E_{v, S e q_{2}}^{m^{\prime}}}
\end{aligned}
$$

Step 24: Determine fault type as follows,

$$
K_{2}^{m^{\prime}}<K_{2}^{T H}, K_{3}^{m^{\prime}}<K_{31}^{T H} \quad \Rightarrow \quad \text { double phase fault }
$$




$$
\begin{array}{ccc}
K_{2}^{m^{\prime}}<K_{2}^{T H}, K_{3}^{m^{\prime}}>K_{31}^{T H} & \Rightarrow & \text { three phase fault } \\
K_{2}^{m^{\prime}}>K_{2}^{T H}, K_{3}^{m^{\prime}}<K_{32}^{T H} & \Rightarrow & \text { single phase fault } \\
K_{2}^{m^{\prime}}>K_{2}^{T H}, K_{3}^{m^{\prime}}>K_{32}^{T H} & \Rightarrow & \text { double phase to ground } \\
& \text { fault }
\end{array}
$$

Step 25: determine the engaged phases in the fault using the energy values of the $a, b$, and $c$ phase current waveforms, knowing that the faulty phases have larger energy values.

Subroutine 7: Calculating reliability indices

Step 26: Execute load flow and calculate and save not-supplied energy.

Step 27: If simulation state relates to a transient state, go Step 6. Otherwise, go step 28.

Step 28: If simulation time is lower than 8760 hours (one year), go Step 1; otherwise calculate the EENS.

Step 29: Compare simulation time with 1000 years. If lower than this value, go subroutine1; otherwise calculate ENS and percentage of successful performances as the final result.

Based on the mentioned steps, implementing of the proposed method is shown in Figure 4.

\section{SimUlation RESUlts}

To evaluate the proposed method, it was implemented on a sample microgrid which is presented in Figure 5 having the substructure pointed earlier, the same to the one used in [18]. This microgrid is connected to upstream network through Bus 1. Four DGs are connected in buses 4, 5, 6, and 9. Specifications for network load are presented in Table I. Based on the Table I, loads are categorized into three types of residential, commercial and industrial. Blackout cost for each load is extracted from [19]. In addition, daily load curve for loads is based on the patterns in [20]. Considering the dynamic state of microgrid topology, like study [20] it was supposed that DGs in each feeder connect to the network when the feeder load reaches $97 \%$ load peak.

\section{TABLE I. CHARACTERISTICS OF THE NETWORK LOADS}

\begin{tabular}{|c|c|c|c|}
\hline $\begin{array}{c}\text { Bus } \\
\text { number }\end{array}$ & $\begin{array}{c}\text { Active power } \\
\text { (MW) }\end{array}$ & $\begin{array}{c}\text { Reactive power } \\
\text { (MVAr) }\end{array}$ & Load type \\
\hline 2 & 4.8 & 1.5 & Commercial \\
\hline 3 & 4.5 & 2.5 & Industrial \\
\hline 4 & 2.7 & 0.8 & Municipal \\
\hline 5 & 2.5 & 0.5 & Municipal \\
\hline 6 & 2.2 & 0.7 & Industrial \\
\hline 7 & 3.4 & 4 & Commercial \\
\hline 8 & 2 & 1 & Municipal \\
\hline 9 & 3 & 1 & Municipal \\
\hline
\end{tabular}

For simplicity of calculations, it was assumed that all lines have the same repair and failure rates, and their values were 3 hours per fail and 0.8 fails per year respectively. It is worth noting that DGs' reliability assumed $100 \%$. The system studied is analyzed in two different scenarios. In the first scenario, it is assumed that fault is detected using traditional methods (high current value of a threshold value) by the central server. In the second scenario, according to the algorithm, the new method is used for fault detection. The results of these scenarios are then compared. It is worth noting that ENS reliability index and percentage of successful performance of protection system calculated and analyzed for both scenarios. In both scenarios, transient states resulted from entering and exiting DGs and entering lines are assumed as the transient states. It is necessary that the proposed method does not react for these transient states because some of loads will not be power supplied due to the wrong reaction of the protection system.

As the results in Table II represent, the proposed method has more appropriate indices for fault detection than those of the traditional methods. The reason is that the new methods have extraordinary accuracy in detecting faults with limited current. See Table III for more explanation. According to this table, while microgrid is working on isolated mode and only DG1 exists in the network, a fault in line 7 occurs. As it is clear, since one protected setup can be saved, therefore in the traditional methods, covering all faults is not possible. This issue is clear in Table III; while module 6 has been set up on $4 \mathrm{kA}$ performance current, and only DG1 exists in the network, the provided fault current by this DG will be $1.96 \mathrm{kA}$. Therefore, the fault is not detected by the main protection system and this issue will increase power outages. That's why in Table II both ENS index and percentage of successful performance of the proposed method are higher than that those of the traditional methods of fault detection. To do so, the behavior of proposed method versus double phase to ground fault, $\mathrm{F}_{1}$, on phases $a$ and $b$ with $100 \Omega$ fault resistance on line 2 of sample microgrid of Figure 5 will be analyzed.

To determine the values of the energy and the STD thresholds of voltage and current signals for zero, positive and negative sequences, the worst situations that could challenge the proposed method which may interrupt the performance of detection algorithm are considered. These worst case scenarios are determined in such a way that fault detection algorithm can detect all the occurred faults and become indifferent for the transient stable states occurred in the network. The values for the normalized energy thresholds and also the normalized STD thresholds for the voltage and current signals for zero, positive and negative sequences are presented in Table IV and also based on the network topology, all possible fault types in various locations of the microgrid are simulated and the values of $K_{2}^{T H}, K_{31}^{T H}$, and $K_{32}^{T H}$ are found to be $0.458,0.465$, and 1.221 , respectively.

Table $\mathrm{V}$ includes the values for the normalized energy and the standard deviation for zero and negative sequences of voltage and current signals obtained from S-transform for all modules after fault $F_{1}$ is occurred. Based on Table $V$, fault occurrence is detected by $1,2,3,5$, and 6 modules. To find out the location of fault, Table VI includes energy values of zero, positive and negative sequences of current waveforms for the modules which detected the fault. Based on Table VI, it is observed that the values of $K_{1}$ at sensing modules $m=2$ and 3 are greater than the other ones and based on algorithm of fault location in Figure 4, the detection algorithm has correctly detected the fault location on Line 2. 


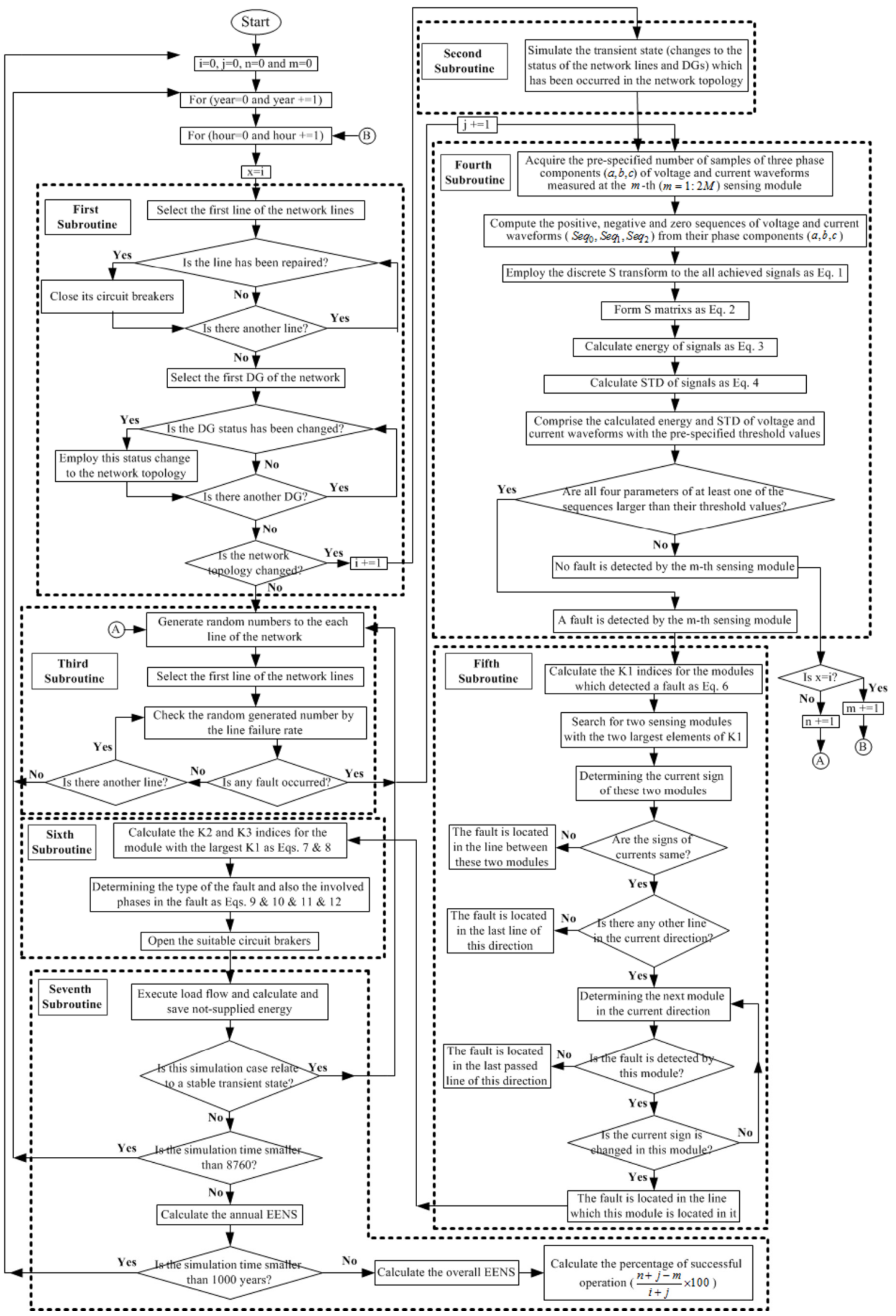

Fig. 4. Evaluation algorithm of the proposed method using Mont Carlo simulation 
TABLE II. RELIABILITY INDICES USING THE PROPOSED METHOD

\begin{tabular}{|c|c|c|}
\hline & $\begin{array}{c}\text { Traditional } \\
\text { scenario }\end{array}$ & $\begin{array}{c}\text { The new proposed } \\
\text { method }\end{array}$ \\
\hline ENS (MWh/yr) & 213000 & 113400 \\
\hline $\begin{array}{c}\text { The number of occurred } \\
\text { faults }\end{array}$ & 7459 & 8010 \\
\hline $\begin{array}{c}\text { The number of stable } \\
\text { transient states }\end{array}$ & 14746 & 15301 \\
\hline $\begin{array}{c}\text { Percentage of successful } \\
\text { Operation }\end{array}$ & 51.963 & 99.438 \\
\hline
\end{tabular}

TABLE III. FAULT EVALUATION USING TRADITIONAL METHODS

\begin{tabular}{|c|c|c|c|}
\hline Fault location & Fault current & Threshold magnitude & Fault detection \\
\hline Line 7 & $1.96 \mathrm{kA}$ & $4 \mathrm{kA}$ & Unsuccessful \\
\hline
\end{tabular}

TABLE IV. NORMALIZED THRESHOLD VALUES

\begin{tabular}{|c|c|c|c|}
\hline Parameter & Magnitude & Parameter & Magnitude \\
\hline$\overline{E_{i, S e q_{0}}^{T H}}$ & 0.045428 & $\overline{S T D_{i, S e q_{0}}^{T H}}$ & 0.028683 \\
\hline$\overline{E_{i, S e q_{1}}^{T H}}$ & 0.128013 & $\overline{S T D_{i, S e q_{1}}^{T H}}$ & 0.044978 \\
\hline$\overline{E_{i, S e q_{2}}^{T H}}$ & 0.061538 & $\overline{S T D_{i, S e q_{2}}^{T H}}$ & 0.029851 \\
\hline$\overline{E_{v, S e q_{0}}^{T H}}$ & 0.032678 & $\overline{S T D_{v, S e q_{0}}^{T H}}$ & 0.047726 \\
\hline$\overline{1 / E_{v, S e q_{1}}^{T H}}$ & 0.07199 & $\overline{S T D_{v, S e q_{1}}^{T H}}$ & 0.077512 \\
\hline$\overline{E_{v, S e q_{2}}^{T H}}$ & 0.040853 & $\overline{S T D_{v, S e q_{2}}^{T H}}$ & 0.060298 \\
\hline
\end{tabular}

TABLE V. OBTAINED NORMALIZED MAGNITUDES BY DIFFERENT MODULES AFTER OCCURRED FAULT F $F_{1}$

\begin{tabular}{|c|c|c|c|c|c|c|c|c|}
\hline \multirow{3}{*}{$\begin{array}{l}\text { Module } \\
\text { number }\end{array}$} & \multicolumn{4}{|c|}{ Voltage signal } & \multicolumn{4}{|c|}{ Current signal } \\
\hline & \multicolumn{2}{|c|}{ Energy } & \multicolumn{2}{|c|}{ STD } & \multicolumn{2}{|c|}{ Energy } & \multicolumn{2}{|c|}{ STD } \\
\hline & $\begin{array}{l}\text { Negative } \\
\text { sequence }\end{array}$ & Zero sequence & $\begin{array}{l}\text { Negative } \\
\text { sequence }\end{array}$ & Zero sequence & $\begin{array}{l}\text { Negative } \\
\text { sequence }\end{array}$ & Zero sequence & $\begin{array}{l}\text { Negative } \\
\text { sequence }\end{array}$ & Zero sequence \\
\hline 1 & 0.165176 & 0.093733 & 0.063072 & 0.159037 & 0.09009 & 0.123389 & 0.039851 & 0.379362 \\
\hline 2 & 0.595129 & 0.247754 & 0.142548 & 0.216125 & 0.195613 & 0.353886 & 0.113859 & 0.781557 \\
\hline 3 & 0.383602 & 0.1834 & 0.084298 & 0.238648 & 0.13365 & 0.220038 & 0.072361 & 0.147892 \\
\hline 4 & 0.102636 & 0.118865 & 0.105177 & 0.213662 & 0.058979 & 0.042004 & 0.020524 & 0.032486 \\
\hline 5 & 0.156191 & 0.036288 & 0.070036 & 0.183455 & 0.097953 & 0.093558 & 0.03576 & 0.145174 \\
\hline 6 & 0.050916 & 0.063322 & 0.045436 & 0.048201 & 0.08735 & 0.075094 & 0.032323 & 0.048626 \\
\hline 7 & 0.026994 & 0.025332 & 0.028792 & 0.041121 & 0.040954 & 0.016833 & 0.013267 & 0.020046 \\
\hline 8 & 0.016614 & 0.009474 & 0.020386 & 0.035604 & 0.022525 & 0.007304 & 0.00498 & 0.012456 \\
\hline
\end{tabular}

TABLE VI. CALCULATED MAGNITUDES OF DIFFERENT PARAMETERS BY MODULES WHICH DETECT THE OCCURRED FAULT $F_{1}$

\begin{tabular}{|c|c|c|c|}
\hline $\begin{array}{c}\text { Module } \\
\text { number }\end{array}$ & $\begin{array}{c}\text { Energy of positive sequence of } \\
\text { current waveform }\end{array}$ & $\mathbf{K}_{\mathbf{1}}$ & $\begin{array}{c}\text { Current } \\
\text { sign }\end{array}$ \\
\hline 1 & 0.133308 & 0.470175 & + \\
\hline 2 & 0.244677 & 1.148062 & + \\
\hline 3 & 0.165693 & 0.739419 & - \\
\hline 5 & 0.09323 & 0.378299 & + \\
\hline 6 & 0.081567 & 0.319105 & + \\
\hline
\end{tabular}

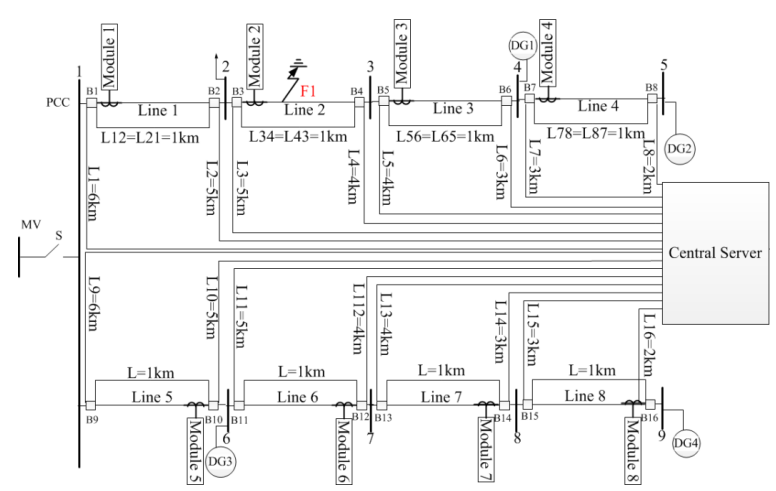

Fig. 5. The microgrid studied

By comparing the above values with the values of $K_{2}^{T H}=0.476 \quad, \quad K_{32}^{T H}=1.232 \quad$ and using $\overline{E_{i, a}^{2}}=0.674645$, $\overline{E_{i, b}^{2}}=0.783688$, and $\overline{E_{i, c}^{2}}=0.341458$, based on (8), it is concluded that the occurred fault type is double phase to ground on phases $a$ and $b$. To determine the fault type, using the values of
$\overline{E_{i, S e q_{0}}^{2}}=0.353886, \quad \overline{E_{i, S e q_{1}}^{2}}=0.244677 \quad, \quad \overline{E_{i, S e q_{2}}^{2}}=0.195613$, $\overline{E_{v, S e q_{0}}^{2}}=0.247754, \overline{1 / E_{v, S e q_{1}}^{2}}=0.468523$, and $\overline{E_{v, S e q_{2}}^{2}}=0.595129$, the values of fault detection indices of $K_{2}^{2}=0.60164$ and $K_{3}^{2}=1.503942$ are calculated. It is worth noting that Figure 6 shows the way of convergence in ENS reliability index for both methods. As it is clear, Monte Carlo algorithm reaches convergence after 1000 years in both methods and the proposed method created better ENS value than traditional methods. This issue shows that the reliability of the proposed method is strongly higher than traditional methods. It is necessary to note that, in addition to the particular accuracy of the presented method in detecting various permanent network faults, the results show that this method has had the appropriate reaction toward the transient states. The percentage of successful Operations in Table II represents the appropriate performance of the methods for both scenarios of permanent and transient faults occurred.

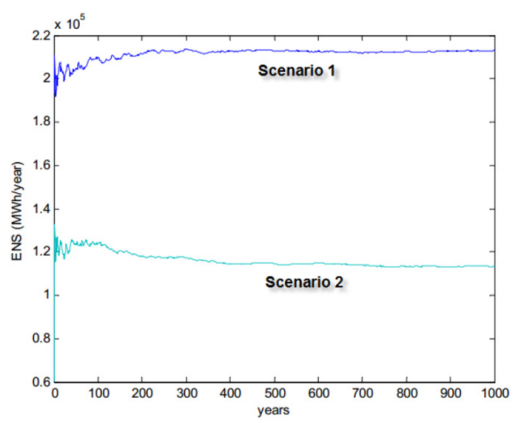

Fig. 6. Monte Carlo simulation convergence in both proposed scenarios 


\section{CONCLUSIONS}

Since the most important challenge of developing microgrids is the matter of fault detection, the scope of this paper is to present a new method for fault detection. The new proposed method uses the signal's energy and STD which has been derived form S-transform. To evaluate the method and determine its performance accuracy versus traditional methods, Monte Carlo simulation was used. Using this simulation, ENS reliability index and performance percentage of proposed method was compared with those of the traditional methods. The evaluations show that the proposed method has strongly higher reliability in contrast to reliability obtained by the transitional fault detection methods. Correct performance of the proposed method was $99.44 \%$ in contrast to the $51.96 \%$ correct performance for traditional methods which represents a significant increase. It is worth noting that the performance accuracy of the intended methods is measured based on their proper reaction against permanent faults and the transient states. Being independent from the topology is one of the advantages of the proposed method for fault detection, since the most important challenge in past designs was their severe dependence on the network topology. Therefore, by using the new proposed method, microgrids can be protected in different topologies with very high reliability.

\section{REFERENCES}

[1] T. Liang, C. Schwaegerl, S. Narayanan, Z. Jian Hui, "From laboratory Microgrid to real markets - Challenges and opportunities", IEEE 8th International Conference on Power Electronics and ECCE Asia (ICPE \& ECCE), pp. 264-271, 2011

[2] T. S. Ustun, C. Ozansoy, A. Zayegh, "Modeling of a Centralized Microgrid Protection System and Distributed Energy Resources According to IEC 61850-7-420", IEEE Transactions on Power Systems, Vol. 27, pp. 1560-1567, 2012

[3] W. Jiang, Z.-y. He, Z. Bo, "The Overview of Research on Microgrid Protection Development", International Conference in Intelligent System Design and Engineering Application, pp. 692-697, 2010

[4] A. A. Memon and K. Kauhaniemi, "A critical review of AC Microgrid protection issues and available solutions", Electric Power Systems Research, Vol. 129, pp. 23-31, 2015

[5] Z. Kai-Hui, X. Ming-Chao, "Impacts of microgrid on protection of distribution networks and protection strategy of microgrid", International Conference on in Advanced Power System Automation and Protection (APAP), pp. 356-359, 2011

[6] M. A. Redfern and H. Al-Nasseri, "Protection of micro-grids dominated by distributed generation using solid state converters", 9th International Conference on Developments in Power System Protection, pp. 670-674, 2008

[7] S. A. Hosseini, H. A. Abyaneh, S. H. H. Sadeghi, F. Razavi, A. Nasiri, "An overview of microgrid protection methods and the factors involved", Renewable and Sustainable Energy Reviews, Vol. 64, pp. 174-186, 2016

[8] P. Basak, S. Chowdhury, S. Halder nee Dey, S. P. Chowdhury, "A literature review on integration of distributed energy resources in the perspective of control, protection and stability of microgrid", Renewable and Sustainable Energy Reviews, Vol. 16, pp. 5545-5556, 2012

[9] H. Al-Nasseri, M. A. Redfern, F. Li, "A voltage based protection for micro-grids containing power electronic converters", IEEE Power Engineering Society General Meeting, 2006

[10] S. A. Hosseini, H. Askarian Abyaneh, S. H. H. Sadeghi, F. Razavi, M. Karami, "Presenting a new method for identifing fault location in microgrids, using harmonic impedance", Iranian Journal of Science and Technology, Vol. 39, pp. 167-182, 2015

[11] A. M. El-Zonkoly, "Fault diagnosis in distribution networks with distributed generation", Electric Power Systems Research, Vol. 81, pp. 1482-1490, 2011

[12] S. A. Saleh, R. Ahshan, M. A. Rahman, M. S. A. Khaizaran, B. Alsayed, "Implementing and testing d-q WPT-based digital protection for microgrid systems", IEEE Industry Applications Society Annual Meeting (IAS), pp. 1-8, 2011

[13] S. R. Samantaray, G. Joos, I. Kamwa, "Differential energy based microgrid protection against fault conditions", IEEE PES in Innovative Smart Grid Technologies (ISGT), pp. 1-7, 2012

[14] H. Al-Nasseri and M. A. Redfern, "Harmonics content based protection scheme for Micro-grids dominated by solid state converters", 12th International Middle-East in Power System Conference, pp. 50-56, 2008

[15] M. Petit, X. Le Pivert, L. Garcia-Santander, "Directional relays without voltage sensors for distribution networks with distributed generation: Use of symmetrical components", Electric Power Systems Research, Vol. 80, pp. 1222-1228, 2010

[16] M. A. Zamani, T. S. Sidhu, A. Yazdani, "A protection strategy and microprocessor-based relay for low-voltage microgrids", IEEE Transactions on Ppower Delivery, Vol. 26, pp. 1873-1883, 2011

[17] R. Billinton, A. Sankarakrishnan, "A comparison of Monte Carlo simulation techniques for composite power system reliability assessment", IEEE Conference Proceedings on Communications, Power, and Computing in Wescanex 95, Vol. 1, pp. 145-150, 1995

[18] W. K. A. Najy, H. H. Zeineldin, W. L. Woon, "Optimal protection coordination for microgrids with grid-connected and islanded capability", IEEE Transactions on Industrial Electronics, Vol. 60, pp. 1668-1677, 2013

[19] G. Wacker, R. Billinton, "Customer cost of electric service interruptions”, IEEE Proceedings, Vol. 77, pp. 919-930, 1989

[20] M. Gilvanejad, H. A. Abyaneh, K. Mazlumi, "Estimation of the overload-related outages in distribution networks considering the random nature of the electrical loads", IET Generation, Transmission \& Distribution, Vol. 7, pp. 855-865, 2013 